\title{
An effective bioremediation approach for enhanced microbial degradation of the veterinary antibiotic sulfamethazine in an agricultural soil
}

\author{
Natalie Hirth ${ }^{1} \mathbb{D}$, Edward Topp ${ }^{2}$, Ulrike Dörfler ${ }^{1}$, Erhard Stupperich ${ }^{3}$, Jean Charles Munch ${ }^{4}$ and Reiner Schroll ${ }^{{ }^{*}}$
}

\begin{abstract}
Background: The veterinary antibiotic Sulfamethazine (SMZ) contaminates soils via manure applications. Like other soil contaminants (herbicides, fungicides, and nematicides), it has to be degraded. The main challenge is that SMZ biodegradation with bacteria is impeded, since SMZ is a bacteriostatic antibiotic, designed to block microbes in their growth.

Results: In this study, we enriched the indigenous soil microbial community (including the single strain Microbacterium sp. (448, adapted to SMZ degradation) from a Canadian soil and we present a suitable approach, for soil remediation by inoculating a German soil with this microbial community established on carrier particles, at environmentally relevant concentrations of $1 \mathrm{mg} \mathrm{kg}^{-1}$. When compared with the isolated SMZ-degrading strain (also obtained from Canada), the microbial community outperformed the mineralization rates of the isolated strain in soil. The negligible soil native SMZ mineralization was successfully increased to 44 and $57 \%$ within 46 days, by the microbial community. The sustainability of this increased SMZ mineralization capacity was proven by the rapid mineralization of a second application of ${ }^{14} \mathrm{C}-\mathrm{SMZ} 112$ days after the first.

Conclusions: The pronounced SMZ mineralization and the high amount of non-extractable ${ }^{14} \mathrm{C}$-residues (NER) in the inoculated soil indicate that the NER are mainly of biogenic origin (metabolically fixed ${ }^{14} \mathrm{C}$ ). Therefore, the applied inoculation approach decreased the risk of persistent non-extractable SMZ residues. Together with our former studies, this specific soil inoculation approach was tested for three substances with different physico-chemical properties, indicating that this soil bioremediation technique might also be used for other substances.
\end{abstract}

Keywords: Sulfamethazine, Enhanced biodegradation, Microbial community, Carrier particles, Soil, Remediation

\section{Background}

Sulfamethazine (4-Amino- $N$-(4,6-dimethyl-2-pyrimidinyl) benzenesulfonamide; SMZ) is a veterinary sulfonamide antibiotic used against infections of the respiratory tract, mainly in swine farming $[1,2]$. It hampers the production of folic acid in target microbes. In the USA, no monitoring of the antibiotic consumption is in place, so

\footnotetext{
*Correspondence: schroll@helmholtz-muenchen.de

${ }^{1}$ Research Unit Microbe-Plant Interactions, Helmholtz Zentrum

München, German Research Center for Environmental Health $(\mathrm{GmbH})$,

85764 Neuherberg, Germany

Full list of author information is available at the end of the article
}

reliable data are scarce, and in most cases, they are estimated [3, 4]. In Europe, a cumulative usage of 2855.2 tons of antibiotics considering 7 European countries in 2004 is reported [5]. In Germany, 162 tons of sulfonamides have been sold to veterinarians in the year 2012, which makes them the third most sold group of antibiotics after tetracyclines and penicillin [6].

After administration to farm animals, parent compounds as well as metabolites of the veterinary antibiotics are excreted and reach agricultural soils either directly by grazing animals or via the application of manure to land after a storage period. Haller et al. [2] quantified SMZ in 
the manure of treated pigs and calves of six farms mainly in the range of $\mathrm{mg} \mathrm{kg}^{-1}$. By application of liquid manure to fields, the risk of contaminating other environmental compartments is thus heightened [7,8]. Christian et al. [9] found SMZ residual concentration both in surface water $\left(7 \mathrm{ng} \mathrm{L}^{-1}\right)$ and soil samples $\left(15 \mu \mathrm{g} \mathrm{kg}^{-1}\right.$, dry weight) 7 months after a liquid manure application, indicating a high stability of SMZ in soil. These non-lethal concentrations can select resistant microorganisms $[10,11]$ which can then potentially transfer resistance to other soil bacteria, including human pathogens, via gene transfer [12-16].

To reduce these risks for the environment and human health, an effective long-term approach is strived, to decontaminate soils from SMZ, without destroying soils, instead preserving them for further agricultural usage. To decontaminate soils from antibiotics, which are applied to the fields regularly, the bioremediation approach should not only be efficient, but also sustainable. In this case, "sustainability" means that the microbes should be applied to the soil only once and they should sustain their degradation ability over time and degrade the contaminant again, when next it is applied.

Until now, studies on SMZ removal have been focusing on the decontamination of waste water reactors using activated sludge [17-20] electrochemical SMZ removal from aqueous solutions [21-23], SMZ removal from water and soil using biochar $[24,25]$, gamma irradiation in sewage and aqueous solution [26-28], and other adsorption removal techniques [29]. All of these techniques are not developed for large-scale soil remediation of whole agricultural areas.

Oliveira et al. [20] reported that SMZ degradation was mostly studied in activated sludge systems and anaerobic waste water treatment; nevertheless, information about successful SMZ degradation is limited and where a high success was reported, the study was conducted with disproportional high concentrations of SMZ $\left(90 \mathrm{mg} \mathrm{L}^{-1}\right)$. In their study, SMZ was biodegraded at the environmental concentration of $100 \mu \mathrm{g} \mathrm{L}{ }^{-1}$ in anaerobic conditions. Since it was dependent on the availability of easily degradable organic matter, a cometabolic degradation of SMZ was suggested.

All of these studied techniques focused on waste water or sludge and did not consider decontamination of polluted agricultural soils.

The only study showing a metabolic SMZ degradation conducted by indigenous soil microbes was done by Topp et al. [30]. High SMZ degradation by indigenous soil microbes was observed in laboratory experiments after long-term application of SMZ to the field (one time per year for 10 years). Topp et al. found out that for the success of this study, it was necessary to use higher SMZ concentrations than the ones reported in soil $\left(10 \mathrm{mg} \mathrm{kg}^{-1}\right)$.
As the decontamination studies, mentioned before, focused on SMZ degradation in aqueous and sometimes anaerobic systems, we saw the need to provide an approach that effectively accelerates the SMZ mineralization in soil. This approach should be successful at a concentration of $1 \mathrm{mg} \mathrm{kg}^{-1}$ which is close to environmental conditions [2], and should be directly applied to soil and avoid destruction of soil structure and relocation as performed by chemical soil extraction, soil combustion, or other harsh ex situ soil remediation techniques. In this soil inoculation approach, not only a single strain capable to degrade a soil contaminant is applied, but a microbial community. The microbial community was enriched by us from an aliquot of this Canadian soil, from which Topp et al. [30] isolated the single SMZ-degrading strain earlier. Furthermore, this microbial community was attached to a protective material (defined clay particles) to improve the survival of the microbes in the new and foreign soil environment. We already presented this very efficient approach, for enhanced biodegradation of other organic chemicals in soils, which ensures that the introduced function of accelerated mineralization survives and establishes in the new soil environment [31-33]. This time, we successfully applied the approach to even decontaminate soil from an antibiotic, which makes it more difficult for the microbial community to survive and support the degrading strain, because all microbes that are sensitive are affected by the SMZ application.

The aim of this study was to apply this soil inoculation approach, for the successful mineralization of an antibiotic, SMZ, in soils. Our objective was, therefore, to enrich the microbial community, which also includes the degrading strain, from SMZ contaminated soil, establish it on carrier particles, introduce it to another ${ }^{14} \mathrm{C}-\mathrm{SMZ}$ contaminated soil, and quantify the SMZ mineralization via trapping of ${ }^{14} \mathrm{CO}_{2}$.

\section{Methods}

\section{Chemicals}

Uniformly, $\quad{ }^{14} \mathrm{C}$-ring-labeled sulfamethazine (4-Amino- $N$-(4,6-dimethyl-2-pyrimidinyl) benzenesulfonamide, SMZ, CAS Number: 57-68-1) was purchased from Campro Scientific GmbH (Veenendaal, The Netherlands) with a specific radioactivity of $673.4 \mathrm{MBq} \mathrm{mmol}^{-1}$, and a radiochemical purity of $>98 \%$. Non-labeled SMZ was obtained from Sigma Aldrich (Taufkirchen, Germany). The ${ }^{14} \mathrm{C}$-SMZ was blended with non-labeled SMZ to produce the ${ }^{14} \mathrm{C}-\mathrm{SMZ}$ application standards (spec. radioactivities ranging from 80 to $500 \mathrm{MBq} \mathrm{mmol}^{-1}$ ). Carbo-Sorb ${ }^{\circledR} E$ and the scintillation cocktails (Permafluor ${ }^{\circledR} \mathrm{E}+$, Ultima Gold ${ }^{\mathrm{TM}} \mathrm{XR}$ and Ultima $\mathrm{Flo}^{\mathrm{TM}}$ ) were purchased from PerkinElmer (Waltham, USA). All 
other chemicals and solvents were purchased in analytical grade from Merck (Darmstadt, Germany).

\section{Soils}

Two soils (Hohenwart and Scheyern1) were sampled from agricultural fields in Germany $(0-10 \mathrm{~cm}$ depth), air dried, sieved $(<2 \mathrm{~mm})$, and stored at $-20{ }^{\circ} \mathrm{C}$. Prior to each experiment, the soils were thawed at $4{ }^{\circ} \mathrm{C}$ for 1 week, then moistened to a water tension of $-15 \mathrm{kPa}$ [34] and equilibrated at room temperature $\left(20 \pm 1{ }^{\circ} \mathrm{C}\right)$ for another week to gently activate the native soil microorganisms from the frozen state. None of the soils had the ability to degrade SMZ naturally.

Soil Hohenwart (13\% clay, $19 \%$ silt, $68 \%$ sand, $1.0 \%$ organic carbon, $0.1 \%$ total $\mathrm{N}, \mathrm{pH} 6.7,48.600^{\circ}$ latitude, $11.383^{\circ}$ longitude, $392 \mathrm{~m}$ altitude) was used for the experiments stimulating the indigenous soil bacteria to degrade SMZ by applying it in different concentrations and frequencies ("adaptation experiments"). In the last 10 years before sampling, this soil was not treated with pesticides or antibiotics and received a barley, maize, and wheat crop rotation, and finally, the field was let to rest for three years.

Soil Scheyern1 (sandy loam: $20 \%$ clay, $40 \%$ silt, $40 \%$ sand, $1.5 \%$ organic carbon, $0.2 \%$ total $\mathrm{N}, \mathrm{pH} 7.8,48.493^{\circ}$ latitude, $11.432^{\circ}$ longitude, $497 \mathrm{~m}$ altitude) was inoculated with the microbial community for the enhanced SMZ mineralization. This soil was sampled on the agricultural research farm Scheyern in Germany. It has an organic farming crop rotation containing potatoes, winter wheat, sunflower, winter rye, and grass-clover-alfalfa as intertillage and cover crop.

The Canadian soil aliquot, where we enriched the microbial community (including the SMZ-degrading strain) from, was sent to us from Canada and is described precisely in the paper of Topp et al. [30]. The two soils only used for the in situ bioavailability experiment are described in the Additional file 1: Table S1.

\section{In situ bioavailability of SMZ}

To investigate the in situ bioavailable ${ }^{14} \mathrm{C}-\mathrm{SMZ}$ over time in soil, the ${ }^{14} \mathrm{C}$-radioactivity in the soil pore water of three different soils (Scheyern1, Scheyern2, and Neumarkt) was determined according to Folberth et al. [35] over a period of 21 days. The characteristics of all 4 soils used in this work are described in the Additional file 1: Table S1. The three soils (35 g dry weight equivalent) were spiked with aqueous ${ }^{14} \mathrm{C}$-SMZ application solution $\left(1 \mu \mathrm{g} \mathrm{g}^{-1}\right)$ and incubated in $250 \mathrm{ml}$ amber bottles (30 replicates). For spiking the soil, $200 \mu \mathrm{l}$ of ${ }^{14} \mathrm{C}$-SMZ application standard was applied dropwise to a $5 \mathrm{~g}$ oven dried $\left(105^{\circ} \mathrm{C}, 24 \mathrm{~h}\right)$ soil aliquot and carefully mixed. The spiked aliquot was transferred to fresh, equilibrated soil (30 g, dry weight) in the amber bottle, and thoroughly stirred with a spatula. The soil was then adjusted to a density of $1.3 \mathrm{~g} \mathrm{~cm}^{-3}$ and a soil water tension of $-15 \mathrm{kPa}$ [34]. The incubators were closed with parafilm and stored in a desiccator with water at the bottom to prevent drying of the samples. After different incubation times $(1,2,3,4,7,8$, $9,10,14$, and 21 days), three replicates of each soil were subjected to centrifugation to determine the current in situ bioavailability of SMZ. For this purpose, $30 \mathrm{~g}$ (dry weight) of the incubated soil were centrifuged for $90 \mathrm{~min}$ at $9000 \mathrm{rpm}$ and $20^{\circ} \mathrm{C}$. After centrifugation, two aliquots of $1 \mathrm{ml}$ of the soil pore water were each mixed with $4 \mathrm{ml}$ Ultima Gold XR and the ${ }^{14} \mathrm{C}$-radioactivity was measured in a liquid scintillation counter (Tricarb 2800TR, PerkinElmer, Waltham, USA). The amount of ${ }^{14} \mathrm{C}$ extracted from the soil pore water was calculated as percentage of the applied ${ }^{14} \mathrm{C}-\mathrm{SMZ}$ and was considered to be the amount of SMZ, available for in situ biodegradation by soil microbes.

\section{Adaptation of soil microorganisms to SMZ degradation}

To stimulate the adaptation process of native soil microbes under laboratory conditions, different experimental soil environment scenarios were applied to soil Hohenwart. No microbes were added to soil, only SMZ was applied at the start of the scenarios, to stimulate the indigenous soil microorganisms to degrade SMZ. The soil was incubated at constant soil water tension $(-15 \mathrm{kPa})$, at fluctuating soil water tension (drying and rewetting cycles with a range from -15 to $-700 \mathrm{kPa}$ ) and at different soil temperatures, achieved by storing soil incubators at room temperature $\left(20 \pm 1^{\circ} \mathrm{C}\right)$ and in a water bath $\left(30 \pm 1^{\circ} \mathrm{C}\right)$. These different scenarios were conducted in two different SMZ concentrations (10 and $100 \mu \mathrm{g} \mathrm{g}^{-1}$ ) and some variants received eight further SMZ applications. The incubation time lasted 160 days. Two samples with $100 \mu \mathrm{g} \mathrm{g}^{-1} \mathrm{SMZ}$, two samples with $10 \mu \mathrm{g} \mathrm{g}^{-1} \mathrm{SMZ}$, and two samples where SMZ was applied several times at $5 \mu \mathrm{g} \mathrm{g}^{-1}$ were incubated longer (430 days) to enhance the chance for adaptation.

Each of the scenarios was conducted with $35 \mathrm{~g}$ (dry weight) of soil Hohenwart. SMZ was applied to the soil according to the procedure described in "In situ bioavailability of SMZ" section and the soil samples were incubated in the dark at a soil density of $1.3 \mathrm{~g} \mathrm{~cm}^{-3}$. Soil samples for the approaches with fluctuating soil water tension were placed in $100 \mathrm{ml}$ flasks, moistened to a water tension of $-15 \mathrm{kPa}$, connected to a laboratory $\mathrm{CO}_{2}$-trapping system by Lehr, Scheunert [36] (modified), and dried for 7 days to a water tension of $-700 \mathrm{kPa}$ by continuously passing dried air through the system $\left(1.3 \mathrm{l} \mathrm{h}^{-1}\right)$. After the drying cycle, the soil samples were remoistened to $-15 \mathrm{kPa}$ and the next drying cycle was 
started. Soil samples with constant soil water tension were incubated in $250 \mathrm{ml}$ amber glass bottles at a water tension of $-15 \mathrm{kPa}$ in the dark. To survey the effect of the exposition scenarios, the mineralization of ${ }^{14} \mathrm{C}-\mathrm{SMZ}$ was quantified in the different treatments, by determining the evolved ${ }^{14} \mathrm{CO}_{2}$.

\section{Enrichment and cultivation of SMZ-degrading bacteria}

The SMZ-degrading Microbacterium sp. strain C448 was isolated by Topp et al. [30] from a Canadian soil, that was exposed to an annual dose of 1 and $10 \mathrm{mg} \mathrm{kg}^{-1} \mathrm{SMZ}$ for 10 years ( $1 \mathrm{mg} \mathrm{kg}^{-1}$ for 5 years, $10 \mathrm{mg} \mathrm{kg}^{-1}$ for 5 years). This degrading strain was sent to us on agar plates together with an aliquot of this Canadian soil. Hence, in this study, we enriched the microbial community (including the SMZ-degrading Microbacterium sp. strain C448) from this soil.

For enrichment, $2 \mathrm{~g}$ soil material ( $<2 \mathrm{~mm}$, wet weight) was added to $19 \mathrm{ml}$ culture medium plus $1 \mathrm{ml}$ of aqueous ${ }^{14} \mathrm{C}$-SMZ in $100 \mathrm{ml}$ Erlenmeyer flasks $\left(10 \mathrm{mg} \mathrm{L}^{-1}\right)$. The mineral salt medium was prepared according to Topp et al. [30] with the exception that vitamins (biotin and thiamin) and trisodium citrate were excluded. To establish the microbial community on the carrier material, 40 sterilized carrier particles $(2-4 \mathrm{~mm}$ sized clay particles, total $\mathrm{N}$ : 3-8 $\mathrm{mg} \mathrm{L}^{-1}, \mathrm{P}_{2} \mathrm{O}_{5}: 5-10 \mathrm{mg} \mathrm{L}^{-1} ; \mathrm{K}_{2} \mathrm{O}$ : $100-120 \mathrm{mg} \mathrm{L}^{-1}$, expanded for high pore volume (>80\%), fired, Seramis GmbH, Mogendorf, Germany) were added to the liquid culture. To avoid microbial contamination of these liquid cultures, filters $(0.20 \mu \mathrm{m}$, Sartorius, Göttingen, Germany) were installed at the air inlet and outlet of the Erlenmeyer flasks and the liquid cultures were incubated at $75 \mathrm{rpm}$ in the dark at $20{ }^{\circ} \mathrm{C}$. Three times per week, the ${ }^{14} \mathrm{CO}_{2}$ evolved from the mineralization of ${ }^{14} \mathrm{C}-\mathrm{SMZ}$ was quantified. After $6-9$ days of incubation the microbial community, established on carrier particles (MCCP) was transferred to fresh ${ }^{14} \mathrm{C}$-SMZ-spiked liquid medium.

The enriched microbial community from the Canadian soil has been analyzed by isolating the DNA from the carrier particles using the FastDNA ${ }^{\mathrm{TM}}$ SPIN Kit for Soil and the FastPrep ${ }^{\circledR}$ Instrument (MP Biomedicals, Santa Ana, CA, USA). The DNA has been prepared for Illumina sequencing according to the Illumina standard protocol. The results were identified mostly to genus level and are attached in the Additional file 2: Fig S1.

The pure Microbacterium sp. strain C448 from Canada was transferred from the agar plates to the same mineral salt medium containing the sterile carrier particles and the ${ }^{14} \mathrm{C}$-SMZ in the same concentrations, volumes, and filters on both sides of the Erlenmeyer flasks. In total, ten subsequent transfers were carried out for the single strain and the community in liquid cultures.

\section{Soil inoculation with the microbial community compared} with the single strain

Two methods of soil inoculation were compared to identify the more effective in mineralizing SMZ (50 g soil dry weight equivalent, $1 \mathrm{mg} \mathrm{kg}^{-1} \mathrm{SMZ}$ ). The soil Scheyern1 which did not have the native capacity to degrade SMZ was inoculated with the isolated strain established on carrier particles (ISCP), as well as the MCCP, and the mineralization was measured by trapping the ${ }^{14} \mathrm{CO}_{2}$ in the soil incubators ( $250 \mathrm{ml}$ amber bottles) for 49 days.

Two different amounts of inoculum were tested for each variant, to overcome possible differences in the amount of applied degrading bacteria on the $\mathrm{CP}$ and have comparable conditions in the ISCP and MCCP application. Therefore, 5 and 10 carrier particles of MCCP as well as 10 and 20 carrier particles for ISCP were added to the ${ }^{14} \mathrm{C}$-SMZ-spiked soil in triplicates.

Soil samples were incubated in the dark at $20 \pm 1{ }^{\circ} \mathrm{C}$, at a soil density of $1.3 \mathrm{~g} \mathrm{~cm}^{-3}$, and a water tension of $-15 \mathrm{kPa}$ for 49 days. Three times per week, ${ }^{14} \mathrm{CO}_{2}$ was collected and quantified. The control samples did not receive a microbial inoculum.

\section{SMZ mineralization in soil inoculated with the microbial community \\ First phase of SMZ mineralization}

For testing the sustainability of this soil inoculation approach, soil Scheyern1 with the SMZ-degrading MCCP was incubated for 112 days, before SMZ was reapplied to the soil.

Prior to soil inoculation, the liquid cultures containing the microbial community were divided into two groups: one group (three replicates) received a second dose of SMZ $\left(5 \mu \mathrm{g} \mathrm{ml}^{-1}\right)$ after 7 days of incubation (higher initial mineralization activity, HA). Another 8 days later, the soil Scheyern1 was inoculated with the MCCP for testing the enhanced SMZ mineralization. The other group (three replicates, lower initial mineralization activity, LA) did not receive a second SMZ dose and was transferred to the soil after 15 days of inoculation. Before being transferred to the soil, the two groups of MCCP showed different mineralization activities in the liquid cultures: the first group showed a higher $\left(2.3 \pm 0.5 \%\right.$ days $\left.^{-1}\right)$ and the latter one a lower SMZ mineralization rate $\left(1.1 \pm 0.1 \%\right.$ days $\left.^{-1}\right)$ (see Additional file 3: Fig S2 for mineralization rates and Additional file 4: Fig S3 for cumulative mineralization of both groups in the 8 days before soil inoculation). Thereby, we tested two MCCP variants with different initial mineralization activities at the beginning of the soil inoculation experiment.

Six replicates of soil Scheyern1 (35 g, dry weight) were spiked with ${ }^{14} \mathrm{C}-\mathrm{SMZ}\left(1 \mu \mathrm{g} \mathrm{g}{ }^{-1}\right)$ and mixed with 12 particles of MCCP. Three replicates were inoculated 
with MCCPs of the HA treatment and three replicates received MCCPs of the LA treatment to test the effect of different initial activity states of the microbial community on its SMZ mineralization capacity. Soil samples were incubated in the dark at $20 \pm 1{ }^{\circ} \mathrm{C}$, at a soil density of $1.3 \mathrm{~g} \mathrm{~cm}^{-3}$, and a water tension of $-15 \mathrm{kPa}$ for 112 days. Three times per week ${ }^{14} \mathrm{CO}_{2}$ was collected and quantified. The control samples did not receive a microbial inoculum.

\section{Second phase of SMZ mineralization}

At the end of the first phase (after 112 days), a second application of ${ }^{14} \mathrm{C}-\mathrm{SMZ}$ was performed to ensure the stability of the approach and to test the sustainability of the enhanced SMZ mineralization capability of the soil over time. The aqueous ${ }^{14} \mathrm{C}$-SMZ solution $(210 \mu \mathrm{l}$, $1 \mu \mathrm{g} \mathrm{g}^{-1}$ soil) was applied to the soil in the incubators and mixed carefully. The soil was re-adjusted to a density of $1.3 \mathrm{~g} \mathrm{~cm}^{-3}$, moistened to a soil water tension of $-15 \mathrm{kPa}$, and incubated in the dark at $20 \pm 1{ }^{\circ} \mathrm{C}$ for another 49 days. SMZ mineralization was quantified three times per week according to 2.8.2. The controls also received a second ${ }^{14} \mathrm{C}$-SMZ application.

\section{Third phase of SMZ mineralization}

After the sustainability of the inoculation approach was tested, a third phase was conducted, where the carrier particles were separated from the bulk soil, before SMZ was applied for a third time. The 12 carrier particles, transferred to soil in the first phase of the experiment, were taken out of each incubator and transferred to new soil incubators containing freshly equilibrated aliquots of the same soil (Scheyern1). SMZ was applied $\left(1 \mathrm{mg} \mathrm{kg}^{-1}\right)$, and the soil was incubated as in the other two phases. The soil, where the carrier particles were taken out, also received a third SMZ application $\left(1 \mathrm{mg} \mathrm{g}^{-1}\right)$ and was incubated without carrier particles in the same way. All incubators were stored in the dark at $20{ }^{\circ} \mathrm{C}$ and mineralization and water content were measured as in the other two phases.

\section{Measurement of ${ }^{14} \mathrm{CO}_{2}$ in the different incubation systems Soil samples with fluctuating water content}

The following aeration system was used to measure the ${ }^{14} \mathrm{CO}_{2}$ generated in the soil samples of the adaptation experiment with fluctuating soil water tension and in the liquid cultures. The soil samples, incubated in $100 \mathrm{ml}$ glass flasks, were connected to a $\mathrm{CO}_{2}$-trapping system consisting of two wash bottles, each filled with $10 \mathrm{ml}$ of a $0.1 \mathrm{~N} \mathrm{NaOH}$ solution for collecting ${ }^{14} \mathrm{CO}_{2}$ [36] (modified). The soil was aerated continuously $\left(1.3 \mathrm{l} \mathrm{h}^{-1}\right)$ with dried air to enable drying of the soils for the fluctuating soil water tension experiment. The $\mathrm{NaOH}$ solution was exchanged three times per week to quantify the trapped ${ }^{14} \mathrm{CO}_{2}$ by taking an aliquot of $2 \mathrm{ml}$, mixing it with $3 \mathrm{ml}$ Ultima Flo and measuring it in a liquid scintillation counter. The liquid cultures, enriched in Erlenmeyer flasks and incubated on a shaker $(75 \mathrm{rpm})$, were connected to this trapping system and aerated $\left(1.3 \mathrm{l} \mathrm{h}^{-1}\right)$ three times per week for $1 \mathrm{~h}$. After each aeration step, the $\mathrm{NaOH}$ was exchanged and a $2 \mathrm{ml}$ aliquot was measured for radioactivity as described above.

\section{Soil samples with constant water content}

This system was used for soil incubation experiments with a constant soil water tension of $-15 \mathrm{kPa}$, namely the treatments with constant soil water tension of the adaptation experiment ("Adaptation of soil microorganisms to SMZ degradation" section), the IS and CP comparison experiment ("Soil inoculation with the microbial community compared with the single strain" section), and the experiment, where SMZ mineralization was enhanced by inoculating the microbial community ("SMZ mineralization in soil inoculated with the microbial community" section). The soil samples were incubated in $250 \mathrm{ml}$ amber bottles. These bottles were closed with a rubber stopper through which a hollow needle was fed to guarantee a constant oxygen supply. At the atmospheric side, the needle was connected to a soda lime filled syringe for adsorbing atmospheric $\mathrm{CO}_{2}$. At the bottom of the rubber cap, a $25 \mathrm{ml}$ plastic beaker was installed and filled with $9 \mathrm{ml}$ of $0.1 \mathrm{~N} \mathrm{NaOH}$ to trap the ${ }^{14} \mathrm{CO}_{2}$ evolved from ${ }^{14} \mathrm{C}$ SMZ mineralization in the soil. Three times per week, the $\mathrm{NaOH}$ solution was exchanged and an aliquot of $2 \mathrm{ml}$ was taken and mixed with $3 \mathrm{ml}$ Ultima Flo, and the ${ }^{14} \mathrm{CO}_{2}$ was measured in a liquid scintillation counter.

\section{${ }^{14} \mathrm{C}$-mass balances}

At the end of the inoculation experiments, ${ }^{14} \mathrm{C}$-mass balances were calculated. For the experiment where the sustainability of the inoculation approach was tested, soil pore water was extracted to determine the in situ bioavailability of SMZ as described in "In situ bioavailability of SMZ" section. For the inoculation of the community compared with the single strain, pore water was not extracted. Extractable ${ }^{14} \mathrm{C}$-residues in soil were determined by mixing $35 \mathrm{~g}$ (dry weight) soil with $0.5 \mathrm{~g}$ of diatomaceous earth prior to accelerate solvent extraction (ASE 200, Dionex, Dreieich, Germany) with a mixture of methanol and water $(80: 20)$ at $100{ }^{\circ} \mathrm{C}$ and $14 \mathrm{MPa}$. To ensure exhaustive extraction, three sequential extraction steps for three times were conducted. Two aliquots of $1 \mathrm{ml}$ of each extract were mixed with $4 \mathrm{ml}$ Ultima Gold XR and measured by liquid scintillation counting. To quantify the non-extractable ${ }^{14} \mathrm{C}$-residues (NER), soil material after ASE was homogenized and three aliquots (250-300 mg) 
were combusted in a Sample Oxidizer (Packard, Dreieich, Germany). The generated ${ }^{14} \mathrm{CO}_{2}$ was trapped in CarboSorb ${ }^{\circledR} \mathrm{E}$, mixed with Permafluor ${ }^{\circledR} \mathrm{E}+$ and quantified in a liquid scintillation counter.

${ }^{14} \mathrm{C}$ mass balance was calculated based on the initially applied ${ }^{14} \mathrm{C}$-SMZ, including the mineralized ${ }^{14} \mathrm{C}$ amount, the extractable, and the non-extractable ${ }^{14} \mathrm{C}$ amount.

\section{Results}

\section{In situ bioavailability of SMZ in soil Scheyern 1}

In the comparison of three soils, differing in their texture, soil Scheyern1 revealed the highest in situ bioavailability of SMZ, and was, therefore, chosen for soil inoculation experiments. The day after ${ }^{14} \mathrm{C}-\mathrm{SMZ}$ application, $29.4 \%$ of the applied radioactivity was found in the soil pore water of soil Scheyern1 (Additional file 5: Fig S4). Thereafter, the ${ }^{14} \mathrm{C}$ content in the pore water decreased continuously with a rapid reduction until day seven $(6.6 \%)$ and a slighter decrease until day 21 (2.5\%). This indicates that more than $90 \%$ of the in situ available SMZ are adsorbed to the soil matrix during the first week, and after that, less than $10 \%$ of the applied SMZ is present in soil pore water and thus in situ available for biodegradation.

\section{Attempts to adapt indigenous soil microorganisms to SMZ degradation}

It was not possible to accelerate the adaptation process of soil microorganisms and to increase the SMZ mineralization in soil Hohenwart, by any of our tested approaches. It can be summarized that even after an "adaptation" period of 430 days, the cumulative mineralization of SMZ did not exceed $6.9 \%$ of the applied SMZ. The SMZ mineralization rates remained below $0.2 \%$ days $^{-1}$ for more than 400 days of incubation. The longsome natural adaptation process could not be enhanced under the selected laboratory conditions.

\section{Enhanced SMZ mineralization in soil Scheyern1 by inoculation}

\section{Soil inoculation with microbial community compared with isolated strain}

We used an aliquot of the Canadian soil that had been adapted to SMZ biodegradation under field conditions [30], to enrich the microbial community, able to degrade SMZ in our lab. To identify the more efficient variant for SMZ mineralization in soil Scheyern1, soil inoculation with the MCCP was compared with inoculation with the ISCP. In both variants, two different amounts of carrier particles were introduced to soil (ISCP: 10 and $20 \mathrm{CP}$, MCCP: 5 and $10 \mathrm{CP}$ ). Figure 1 shows the different mineralization rates of MCCP and ISCP.

The microbial community was able to mineralize SMZ efficiently and showed higher mineralization rates compared with the isolated strain and a higher cumulative mineralization after 49 days [MCCP, 37 and $39 \%$ of applied SMZ depending on the amount of introduced carrier particles (5 and $10 \mathrm{CP}$ ) compared with ISCP, 8 and $14 \%$ of applied SMZ (10 and $20 \mathrm{CP}$ )]. The measurement on the first day showed similar mineralization rates for the soil inoculated with ISCP $10 \mathrm{CP}$ and MCCP 10 CP. Afterwards, the mineralization rates of both variants of the IS were decreasing rapidly, while the mineralization rates of the $\mathrm{MC}$ were increasing to come to a peak between day 4 and 7 .

\section{Soil inoculation with MCCP to test the sustainability of enhanced SMZ mineralization}

After the inoculation of the MCCP turned out to be the more effective bioremediation technique, when compared with the isolated single strain, we repeated the soil inoculation with this community. A second phase was conducted, to find out if this approach is sustainable and the mineralization capacity is stable in soil over time. Finally, in a third phase, the mineralization site was investigated.

The soil Scheyern1, used in our study for soil inoculation experiments, revealed a native SMZ mineralization rate of $0.03 \%$ days $^{-1}$ (Fig. 2). This low natural SMZ mineralization capacity in soil Scheyern1 was increased considerably by inoculation with the SMZ-degrading MCCP in two different states of activity (MCCP-LA and MCCP-HA). Within 46 days, $44 \%$ of the applied ${ }^{14} \mathrm{C}-\mathrm{SMZ}$ was mineralized in total in the soil inoculated with the MCCP that showed a lower SMZ mineralization activity (MCCP-LA). The SMZ mineralization rate was at its maximum of $5.5 \%$ days $^{-1}$ of the applied SMZ, after 4 days of inoculation (Fig. 2a). When soil Scheyern1 was inoculated with the microbial community of higher SMZ mineralization activity (MCCP-HA), the inoculation effect was even more pronounced, resulting in a cumulative SMZ mineralization of $57 \%$ after 46 days and a maximum SMZ mineralization rate of $12.3 \%$ days $^{-1}$ at day 4 (Fig. 2a).

To test the sustainability of this enhanced SMZ mineralization, 112 days after the first SMZ application, a second SMZ application was conducted. In this second phase, the two groups of inoculants (MCCP-HA and MCCP-LA) showed no substantial differences in their SMZ mineralization activities (Fig. 2b). SMZ mineralization was considerably enhanced compared with the control, although not as high as in the first phase when the MCCP-HA and MCCP-LA were freshly inoculated into the soil. After 46 days, $36 \%$ (MCCP-LA) to $38 \%$ (MCCP$\mathrm{HA})$ of the applied ${ }^{14} \mathrm{C}-\mathrm{SMZ}$ were mineralized and the mineralization rates peaked on day 6 in $3 \%$ days $^{-1}$ (MCCP-LA) and on day 8 in $4 \%$ days $^{-1}$ (MCCP-HA) 


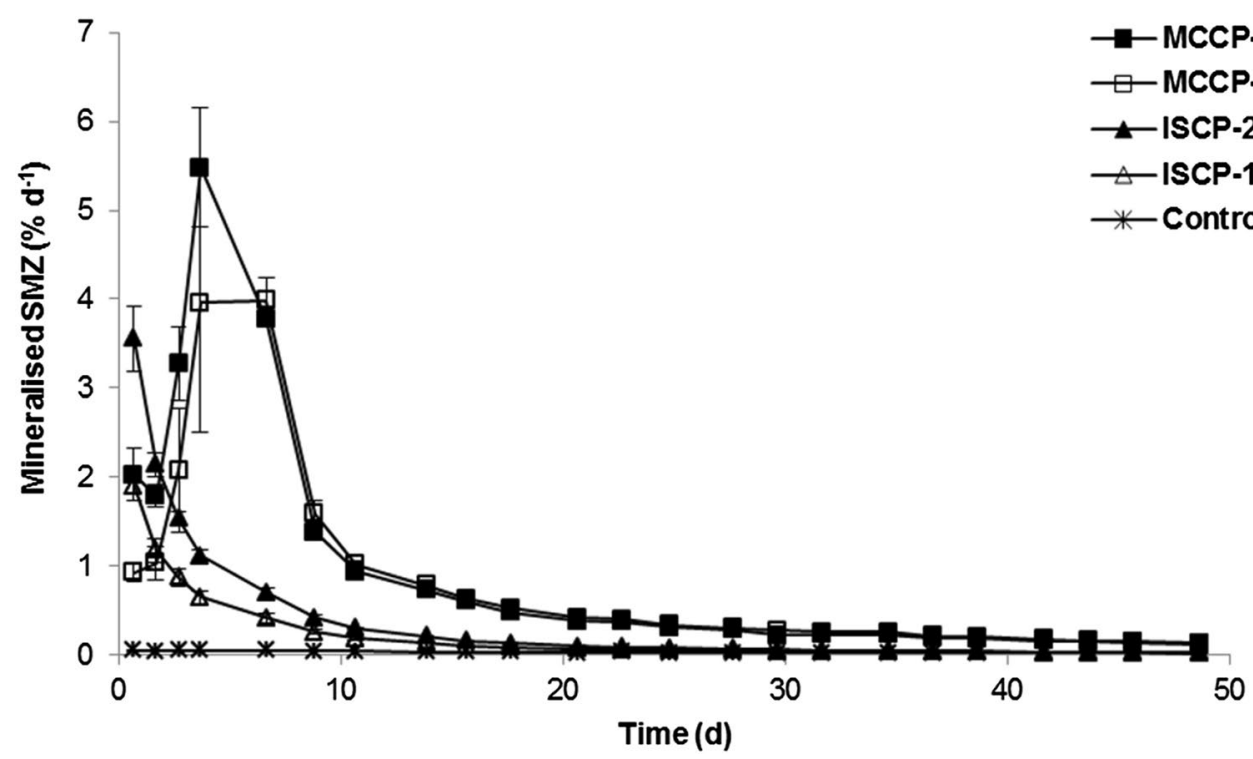

Fig. $1{ }^{14} \mathrm{C}-\mathrm{SMZ}$ mineralisation in soil Scheyern1; comparison of MCCP and ISCP. Mineralization rates of ${ }^{14} \mathrm{C}-\mathrm{SMZ}$ in soil Scheyern1 inoculated with a specific microbial community established on carrier particles (MCCP) and the isolated SMZ-degrading Microbacterium sp. strain C448 on carrier particles (ISCP) quantified via trapping of ${ }^{14} \mathrm{CO}_{2}$ for 49 days. Values represent mean \pm standard deviation; $\mathrm{n}=3$; control signifies "soil without inoculation"

(Fig. 2b). In contrast, the SMZ mineralization rates in the control stayed below $0.1 \%$ days $^{-1}$ and after 46 days, only $1.7 \%$ of the applied ${ }^{14} \mathrm{C}-\mathrm{SMZ}$ was obtained as ${ }^{14} \mathrm{CO}_{2}$.

\section{${ }^{14} \mathrm{C}$-residues in soil}

After the second phase, the soil samples MCCP-HA were analyzed for ${ }^{14} \mathrm{C}$-residues in soil pore water, extractable ${ }^{14} \mathrm{C}$-residues, and non-extractable ${ }^{14} \mathrm{C}$-residues. The ${ }^{14} \mathrm{C}$-residues found in the soil pore water of the inoculated soil samples amounted to $0.5 \%$ (Table 1 ). In the control, $14.2 \%$ of the initially applied radioactivity was found to be methanol-extractable ${ }^{14} \mathrm{C}$ residues, whereas in the inoculated soil samples, the methanol-extractable ${ }^{14} \mathrm{C}$-residues were almost three times lower $(5.1 \%$, Table 1). The inoculated samples also showed lower nonextractable residues $(42.9 \%$, Table 1$)$ compared with the control samples $(81.9 \%$, Table 1$)$. The total recovery of ${ }^{14} \mathrm{C}$-radioactivity was $100.0 \pm 0.6 \%$ and $97.8 \pm 1.1 \%$ in the inoculated and control samples, respectively.

\section{Discussion}

\section{Enhanced mineralization of SMZ in soil by inoculation}

The SMZ-degrading microbial community was enriched on carrier particles, transferred to soil, and was able to mineralize SMZ efficiently. Hence, the microbial degraders survived and established in the new soil environment. Soil Scheyern1 was inoculated with the SMZdegrading microbial community as well as the isolated SMZ-degrading Microbacterium sp. strain C448, both established on carrier particles. The MCCP was able to mineralize SMZ efficiently and showed higher mineralization rates compared with the ISCP and a higher cumulative mineralization. These results lead to the assumption that the microbial community supports the degrading strain. When inoculated as isolated strain, mineralization rates were decreasing from the beginning. This indicates a lower cell activity and maybe also a reduction of cell material of the isolated strain. When inoculated as the whole soil born microbial community, the mineralization rates were increasing which indicates an increasing cell activity and probably also a growth of cells. The MCCP was, therefore, selected for the following experiment where the sustainability of the inoculation approach was tested.

The higher SMZ mineralization capacity of the inoculant MCCP-HA is most probably due to an increased cell growth in the liquid culture induced by a second SMZ dose before the inoculant was transferred from the liquid culture to the soil. Thus, more active cells were introduced into the soil, resulting in a higher initial SMZ mineralization activity. The inoculant MCCP-LA where the cell growth was not stimulated by a second SMZ dose showed a lower initial SMZ mineralization activity in the soil. The reason why the LA group did not attain the SMZ mineralization capacity of the HA group can be explained by the bioavailability of SMZ. The highest amount of SMZ in the soil pore water was found in the first 7 days after application, thereafter, most of the 

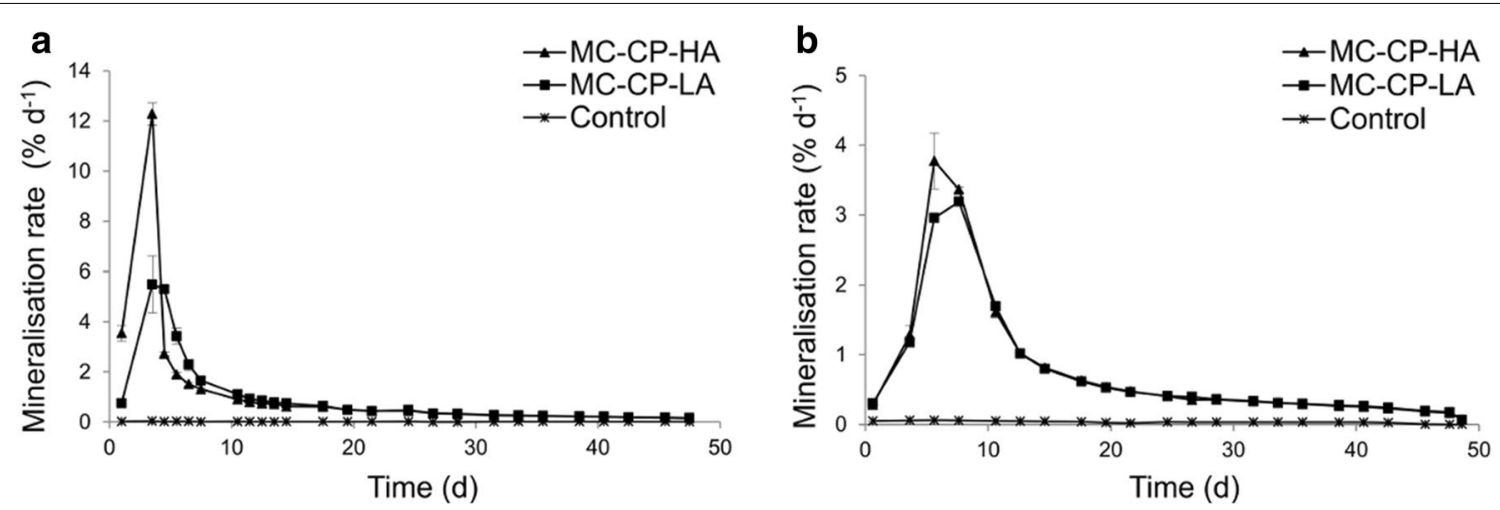

Fig. 2 Mineralisation rates after soil inoculation with MCCP in two consecutive incubation phases. Mineralization rates of ${ }^{14} \mathrm{C}-\mathrm{SMZ}$ in soil Scheyern1 after inoculation with a specific microbial community established on carrier particles (MCCP) quantified via trapping of ${ }^{14} \mathrm{CO}_{2}$. To test the sustainability of the approach, ${ }^{14} \mathrm{C}-\mathrm{SMZ}$ was applied twice. a First, ${ }^{14} \mathrm{C}-\mathrm{SMZ}$ application and incubation for 112 days. For comparison, the graph was cropped at day 50. (Full 112 days are presented in Additional file 6: Fig. S5). b Second, ${ }^{14} \mathrm{C}-\mathrm{SMZ}$ application and ${ }^{14} \mathrm{C}-\mathrm{SMZ}$ mineralisation measurement after 112 days of incubation. Values represent mean \pm standard deviation; $n=3$; control signifies "soil without inoculation"

available SMZ was adsorbed to the soil. For this reason, the MCCP-HA group with its higher initial SMZ mineralization activity had an advantage over the MCCP-LA group: it was able to mineralize more of the available SMZ within the first 7 days - when the SMZ bioavailability was highest-than the MCCP-LA group with its lower initial SMZ mineralization activity. Independent from the differences between the two inoculants, the results present the efficiency of the inoculation approach in enhancing the SMZ mineralization.

\section{Sustainability of the inoculation approach}

After 7 days, most of the SMZ applied has adsorbed to the soil matrix as shown in the bioavailability experiment (Additional file 5: Fig S4), only less than $10 \%$ of the initially applied SMZ is available for rapid degradation. Our data show that even after 112 days, when SMZ was applied again, the SMZ-mineralizing function was still present, which emphasizes the long-term sustainability of the inoculation approach. As the manure is typically applied to the fields twice per year, the veterinary residues are reaching the grounds discontinuously. In between, the bioavailability of SMZ is very low due to sorption processes, and the amount of SMZ is probably not sufficient for energy support of the SMZ-degrading microbes. Hence, they have to survive for a long time under limited SMZ concentrations using other carbon and energy sources, provided by the soil environment. Yet, the bacterial community conserves its SMZ-degrading capability for at least 112 days to restart the degradation process after another SMZ application.

Since there was no difference in the SMZ mineralization capacity of the inoculants MCCP-LA and MCCP-HA after the second SMZ application, it can be concluded that on the long term, the SMZ mineralization capacity of the inoculants mainly depends on the SMZ concentration as compared with its initial SMZ mineralization activity.

The enhanced SMZ mineralization, measured after the first ${ }^{14} \mathrm{C}-\mathrm{SMZ}$ application, as well as after the second application is exclusively due to the inoculated microbial community, since the "adaptation" experiments have clearly excluded adaptation processes as a reason for accelerated SMZ degradation. In a third phase, the carrier particles were separated from the bulk soil, before SMZ was applied for a third time (Additional file 7: Fig S6; Additional file 8: Fig S7). In the bulk soil without carrier particles, $56 \%$ of the applied SMZ was degraded after 62 days. These results reveal that microbes were able to proliferate in the soil matrix and continue the SMZ degradation there, which is a very important factor to ensure the success of this application approach. There is no need to apply the carrier particles again, after the microbes

Table $1{ }^{14} \mathrm{C}$ mass balance at the end of the incubation experiment

\begin{tabular}{llllc}
\hline Treatment & ${ }^{\mathbf{1 4}} \mathbf{C O}_{\mathbf{2}}$ & ${ }^{\mathbf{1 4}} \mathbf{C}$ in soil pore water & ${ }^{{ }^{\mathbf{4}} \mathbf{C} \text { extractable }}$ & ${ }^{\mathbf{1 4} C \text { non-extractable }}$ \\
\hline MCCP-HA & $51.4 \pm 0.3$ & $0.5 \pm 0.0$ & $5.1 \pm 0.0$ & $42.9 \pm 0.8$ \\
Control & $1.8 \pm 0.0$ & not measured & $14.2 \pm 0.5$ & $81.9 \pm 0.5$ \\
\hline
\end{tabular}

Percent distribution and mass balance of ${ }^{14} \mathrm{C}$ in soil Scheyern 1 after 161 days of incubation with MCCP-HA in $\%$ of applied ${ }^{14} \mathrm{C}$-radioactivity. MCCP-HA $=$ Microbial community attached on carrier particles, group of higher initial SMZ mineralization activity; values represent mean \pm standard deviation; $\mathrm{n}=3$ 
have established in the bulk soil. Therefore, we strongly recommend this very effective, sustainable, and stable soil inoculation approach, using a microbial community which supports the degrading strain, and carrier particles for a stable soil inoculation, for remediating soils.

\section{Formation of non-extractable ${ }^{14} \mathrm{C}$-residues (NER)}

In the control samples, the indigenous microbial community was unable to degrade SMZ in a considerable amount and more than $80 \%$ of the applied ${ }^{14} \mathrm{C}$ were found in the NER fraction, which is likely to consist of SMZ and/or SMZ degradation products physico-chemically bound to the soil matrix. The type of binding was not investigated in this study. Several binding mechanisms can play a role in the formation of non-extractable residues, for example, Bialk et al. [37] have proven covalent cross coupling between SMZ and model humic constituents. The rapid formation of high amounts of NER was confirmed by other studies for different sulfonamides [38-40]. Though high affinity to the soil matrix and negligible uptake of sulfonamide-NER by plants and earthworms was shown [39], it cannot be excluded that the NER poses a certain risk for human health, since they can be remobilized during the natural turnover processes of soil organic matter and subsequently contaminate environmental compartments and become bioavailable.

In the inoculated soil samples, the main removal process of SMZ from soil was mineralization, followed by formation of NER. According to the high SMZ mineralization, it can be concluded that the NER in this case are mainly of biogenic origin. Principally, degrading microorganisms can use a xenobiotic as energy and carbon source. When used as carbon source, the degrading bacteria assimilate carbon derived from the xenobiotic to form cellular components, such as sugars, amino acids, etc., and after death of the bacteria, such biomolecules will be fixed in soil organic matter [41]. Thus, the applied inoculation technique enhances the mineralization of SMZ and decreases the risks caused by NER, because they consist to a large amount of biomolecules instead of SMZ or its degradation products.

\section{Résumé of the inoculation approach}

The approach for enhanced mineralization of a soil contaminant by inoculating the soil with a degrading microbial community established on carrier material has already been successfully applied in our previous studies for the herbicide isoproturon and the environmental chemical 1,2,4-TCB [31-33]. Accelerated mineralization of the herbicide isoproturon was shown not only in laboratory experiments, but also under field conditions [31].

This study confirms our hypothesis, as this approach could remarkably enhance the mineralization of the veterinary antibiotic SMZ in soil, despite the considerably low bioavailability of the tested substance SMZ (Additional file 5: Fig S4). The microbial community was successfully established on carrier particles and survived in the new soil habitat, enhancing the SMZ mineralization.

The success of this approach can be mainly explained by three reasons:

1. The results of our former studies [32,33] indicate that the introduced bacteria can establish a protective biofilm on the carrier material. It is known that cells in biofilms have a distinct advantage over submerse bacteria, for example, they have a better chance of survival especially under unfavorable conditions [42-44]. We also found hints for an inter-species communication within the biofilm on the carrier material that promotes the contaminant's mineralization [33].

2. There are strong indications that the contaminant in soils can be transported to the degrading community via diffusion, resulting in mass transfer of the substance to the degradation location on the particles [31].

3. As shown in the present in situ study, this approach is sustainable and the SMZ-degrading function can be established in the new soil environment. Transfer of genetic information from the introduced degraders to the native soil microorganisms is a slow-acting process and happens primarily under high selection pressure, i.e., high concentration of the contaminant, which is normally not the case in agricultural soils. Therefore, it can be assumed that the SMZ-degrading function is performed by the introduced degraders which are able to survive and establish in the new habitat; as has been already verified for the 1,2,4-TCB degrader Bordetella sp. F2 for a time span of 30 days [32].

\section{Conclusions}

Our specific approach of inoculating microbial communities on carrier material was successful for the antibiotic SMZ, as well as for two other substances with different physico-chemical properties and other microbial communities in various soils. As this inoculation approach is successful and sustainable even for SMZ as an antibiotic used against bacteria which is also showing a relatively low bioavailability, we conclude that it is probably also applicable for many other contaminants in soils. Due to the highly enhanced mineralization conducted by the microbial community containing the degrading strain Microbacterium sp. C448, we strongly recommend the utilization of the appropriate microbial community instead of an isolated degrading strain when developing soil remediation methods. 


\section{Additional files}

Additional file 1: Table S1. Soil characteristics of all soils used in this work. The main characteristics, the natural site name and the soil classification are provided, as well as the specific soil water content (\%) equivalent to the soil water tension of pF 2.18 for every soil.

Additional file 2: Fig S2. Gene sequencing of the soil microbial community used for soil inoculation. The composition of the microbial community enriched from soil was analysed by DNA extraction and determination of the $16 \mathrm{~S}$ rRNA-gene sequence by illumina sequencing. The degrading strain Microbacterium sp. C448 can be found within the group of "others", as the DNA of the Microbacteriaceae appeared to less than $1 \%$ of the totally analysed DNA in this sample. Most sequences were analysed to genus level. *Analysis only allows identification on family level. ${ }^{* *}$ Analysis only allows identification on order level. ${ }^{* * *}$ Analysis only allows identification on phylum level.

Additional file 3: Fig S3. Sulfamethazine mineralization rates in liquid cultures. Mineralization rates were measured via ${ }^{14} \mathrm{CO}_{2}$. Bacteria were transferred from liquid culture to soil (on CP) on day 15. The two variants were: $\mathrm{HA}$ : the liquid cultures were incubated with another $5 \mathrm{\mu g} \mathrm{g}^{-1}$ of SMZ on day 8. LA: the liquid cultures did not receive another SMZ application. $n=3$, bars indicate standard deviation.

Additional file 4: Fig S4. Cumulative SMZ mineralization in liquid cultures. The cumulative mineralization was calculated each day from the actual mineralization rates. Bacteria were transferred from liquid culture to soil (on CP) on day 15. The two variants were: HA: the liquid cultures were incubated with another $5 \mathrm{\mu g} \mathrm{g}^{-1}$ of SMZ on day 8. LA: the liquid cultures did not receive another $S M Z$ application. $n=3$, bars indicate standard deviation.

Additional file 5: Fig S5. In situ bioavailability of SMZ in soils. Decrease of in situ bioavailable ${ }^{14} \mathrm{C}-\mathrm{SMZ}$ was measured via soil pore water extraction of three soil types over time.

Additional file 6: Fig S6. Mineralization rates after soil inoculation with MCCP. The ${ }^{14} \mathrm{C}-\mathrm{SMZ}$ mineralization results after the first SMZ application and soil inoculation with microbial community on carrier particles (MCCP), which are also presented in Fig. 2a in the manuscript. Here, the whole incubation time of 112 days is shown. Values represent mean \pm standard deviation; $n=3$; control signifies "soil without inoculation".

Additional file 7: Fig S7. Sulfamethazine mineralization rates in the third phase of soil incubation.After separation of the bulk soil and the carrier particles, ${ }^{14} \mathrm{C}-\mathrm{SMZ}$ was applied again and mineralization rates were measured separately in the two variants. The increase in the mineralization rate in the bulk soil indicates that the degrading bacteria proliferated into the soil. Soil only: soil from phase one and two, where carrier particles have been removed. CP only: the carrier particles, used for inoculation in phases one and two, transferred to fresh soil.

Additional file 8: Fig S8. Cumulative SMZ mineralization in the third phase of soil incubation. The cumulative SMZ mineralization was calculated from the mineralization rates every day. The fast and high SMZ mineralization capacity of the bulk soil is demonstrated. Soil only: soil from phase one and two, where carrier particles have been removed. CP only: the carrier particles, used for inoculation in phases one and two, transferred to fresh soil.

\section{Abbreviations}

ISCP: isolated strain established on carrier particles; MCCP: microbial community established on carrier particles; MCCP-HA: microbial community with higher initial mineralization activity in soil; MCCP-LA: microbial community with lower initial mineralization activity in soil; NER: non-extractable ${ }^{14} \mathrm{C}$-residues; SMZ: sulfamethazine.

\section{Authors' contributions}

$\mathrm{NH}$, writing —original draft preparation, conceptualization, project administration and realization. ET, validation, provision of soil samples, and
Microbacterium sp. strain C448. UD, writing-review and editing, validation. ES, supervision, validation. JCM, writing_-review and editing, supervision. RS validation, conceptualization, and supervision. All authors read and approved the final manuscript.

\section{Author details}

${ }^{1}$ Research Unit Microbe-Plant Interactions, Helmholtz Zentrum München, German Research Center for Environmental Health (GmbH), 85764 Neuherberg, Germany. ${ }^{2}$ Agriculture and Agri-Food Canada, London Research and Development Centre, London, ON N5V 4T3, Canada. ${ }^{3}$ Institute of Microbiology and Biotechnology, UIm University, 89069 Ulm, Germany. ${ }^{4}$ Lehrstuhl für Grünlandlehre, Technische Universität München, 85354 Freising, Germany.

\section{Acknowledgements}

We thank Dr. Michael Rothballer for his help in conducting the DNA analysis via illumina sequencing.

\section{Competing interests}

The authors declare that they have no competing interests.

Received: 21 July 2016 Accepted: 12 October 2016

Published online: 22 October 2016

\section{References}

1. Iglesias A, Nebot C, Miranda J, Vázquez B, Cepeda A. Detection and quantitative analysis of 21 veterinary drugs in river water using high-pressure liquid chromatography coupled to tandem mass spectrometry. Environ Sci Pollut Res. 2012;19(8):3235-49.

2. Haller MY, Müller SR, McArdell CS, Alder AC, Suter MJF. Quantification of veterinary antibiotics (sulfonamides and trimethoprim) in animal manure by liquid chromatography_mass spectrometry. J Chromatogr A. 2002;952(1-2):111-20.

3. Sarmah AK, Meyer MT, Boxall ABA. A global perspective on the use, sales, exposure pathways, occurrence, fate and effects of veterinary antibiotics (VAs) in the environment. Chemosphere. 2006:65(5):725-59.

4. Kümmerer K. Antibiotics in the aquatic environment-a review-Part I. Chemosphere. 2009;75(4):417-34.

5. Kools SAE, Moltmann JF, Knacker T. Estimating the use of veterinary medicines in the European union. Regul Toxicol Pharmacol. 2008;50(1):59-65.

6. Germap. German report on the consumption of antimicrobials in human and veterinary medicine. 2012.

7. Jørgensen SE, Halling-Sørensen B. Drugs in the environment. Chemosphere. 2000;40(7):691-9.

8. Winckler C, Grafe A. Use of veterinary drugs in intensive animal production. J Soils Sediments. 2001;1(2):66-70.

9. Christian T, Schneider RJ, Färber HA, Skutlarek D, Meyer MT, Goldbach HE. Determination of antibiotic residues in manure, soil, and surface waters. Acta Hydrochim Hydrobiol. 2003;31(1):36-44.

10. Andersson DI, Hughes D. Evolution of antibiotic resistance at non-lethal drug concentrations. Drug Resist Update. 2012;15(3):162-72.

11. Gullberg E, Cao S, Berg OG, Ilbäck C, Sandegren L, Hughes D, et al. Selection of resistant bacteria at very low antibiotic concentrations. PLoS Pathog. 2011;7(7):e1002158.

12. Kemper N. Veterinary antibiotics in the aquatic and terrestrial environment. Ecol Ind. 2008;8(1):1-13.

13. Baran W, Adamek E, Ziemiańska J, Sobczak A. Effects of the presence of sulfonamides in the environment and their influence on human health. J Hazard Mater. 2011;196:1-15.

14. Finley RL, Collignon P, Larsson DGJ, McEwen SA, Li X-Z, Gaze WH, et al. The scourge of antibiotic resistance: the important role of the environment. Clin Infect Dis. 2013:57(5):704-10.

15. Wellington EMH, Boxall ABA, Cross P, Feil EJ, Gaze WH, Hawkey PM, et al. The role of the natural environment in the emergence of antibiotic resistance in Gram-negative bacteria. Lancet Infect Dis. 2013;13(2):155-65.

16. Gaze WH, Krone SM, Larsson JDG, Li X-Z, Robinson JA, Simonet P, et al. Influence of humans on evolution and mobilization of environmental antibiotic resistome. Emerg Infect Dis J. 2013;19:7. 
17. Ingerslev F, Halling-Sørensen B. Biodegradability properties of sulfonamides in activated sludge. Environ Toxicol Chem. 2000;19(10):2467-73.

18. Pérez S, Eichhorn P, Aga DS. Evaluating the biodegradability of sulfamethazine, sulfamethoxazole, sulfathiazole, and trimethoprim at different stages of sewage treatment. Environ Toxicol Chem. 2005;24(6):1361-7.

19. García-Galán MJ, Rodríguez-Rodríguez CE, Vicent T, Caminal G, Díaz-Cruz MS, Barceló D. Biodegradation of sulfamethazine by trametes versicolor: removal from sewage sludge and identification of intermediate products by UPLC-QqTOF-MS. Sci Total Environ. 2011;409(24):5505-12.

20. Oliveira GHD, Santos-Neto AJ, Zaiat M. Evaluation of sulfamethazine sorption and biodegradation by anaerobic granular sludge using batch experiments. Bioprocess Biosyst Eng. 2016;39(1):115-24.

21. Sopaj F, Oturan N, Pinson J, Podvorica F, Oturan MA. Effect of the anode materials on the efficiency of the electro-Fenton process for the mineralization of the antibiotic sulfamethazine. Appl Catal B Environ. 2016;199:331-41.

22. Barhoumi N, Oturan N, Olvera-Vargas H, Brillas E, Gadri A, Ammar S, et al. Pyrite as a sustainable catalyst in electro-Fenton process for improving oxidation of sulfamethazine. Kinetics, mechanism and toxicity assessment. Water Res. 2016:94:52-61.

23. Saidi I, Soutrel I, Fourcade F, Amrane A, Floner D, Bellakhal N, et al. Flow electrolysis on high surface electrode for biodegradability enhancement of sulfamethazine solutions. J Electroanal Chem. 2013;707:122-8.

24. Vithanage M, Rajapaksha AU, Zhang M, Thiele-Bruhn S, Lee SS, Ok YS. Acid-activated biochar increased sulfamethazine retention in soils. Environ Sci Pollut Res. 2015;22(3):2175-86

25. Rajapaksha AU, Vithanage M, Ahmad M, Seo D-C, Cho J-S, Lee S-E, et al. Enhanced sulfamethazine removal by steam-activated invasive plantderived biochar. J Hazard Mater. 2015;290:43-50.

26. Chu L, Wang J, Liu Y. Degradation of sulfamethazine in sewage sludge mixture by gamma irradiation. Radiat Phys Chem. 2015;108:102-5.

27. Liu Y, Wang J. Degradation of sulfamethazine by gamma irradiation in the presence of hydrogen peroxide. J Hazard Mater. 2013;250-251:99-105.

28. Liu Y, Hu J, Wang J. Fe2+ enhancing sulfamethazine degradation in aqueous solution by gamma irradiation. Radiat Phys Chem. 2014;96:81-7.

29. Qiang Z, Bao X, Ben W. MCM-48 modified magnetic mesoporous nanocomposite as an attractive adsorbent for the removal of sulfamethazine from water. Water Res. 2013;47(12):4107-14.

30. Topp E, Chapman R, Devers-Lamrani M, Hartmann A, Marti R, Martin-Laurent $F$, et al. Accelerated Biodegradation of Veterinary Antibiotics in Agricultural Soil following Long-Term Exposure, and Isolation of a Sulfamethazine-degrading Microbacterium sp. J Environ Qual. 2013;42(1):173-8.

31. Grundmann S, Fuß R, Schmid M, Laschinger M, Ruth B, Schulin R, et al. Application of microbial hot spots enhances pesticide degradation in soils. Chemosphere. 2007;68(3):511-7.
32. Wang F, Dörfler U, Schmid M, Fischer D, Kinzel L, Scherb H, et al. Homogeneous inoculation vs. microbial hot spots of isolated strain and microbial community: what is the most promising approach in remediating 1,2,4 TCB contaminated soils? Soil Biol Biochem. 2010;42(2):331-6.

33. Wang F, Fekete A, Harir M, Chen X, Dörfler U, Rothballer M, et al. Soil remediation with a microbial community established on a carrier: strong hints for microbial communication during 1,2,4-trichlorobenzene degradation. Chemosphere. 2013;92(11):1403-9.

34. Schroll R, Becher HH, Dörfler U, Gayler S, Grundmann S, Hartmann HP, et al. Quantifying the effect of soil moisture on the aerobic microbial mineralization of selected pesticides in different soils. Environ Sci Technol. 2006;40(10):3305-12.

35. Folberth C, Scherb H, Suhadolc M, Munch JC, Schroll R. In situ mass distribution quotient (iMDQ) - a new factor to compare bioavailability of chemicals in soils? Chemosphere. 2009;75(6):707-13.

36. Lehr S, Scheunert I, Beese F. Mineralization of free and cell-wall-bound isoproturon in soils in relation to soil microbial parameters. Soil Biol Biochem. 1996:28(1):1-8.

37. Bialk HM, Simpson AJ, Pedersen JA. Cross-coupling of sulfonamide antimicrobial agents with model humic constituents. Environ Sci Technol. 2005;39(12):4463-73.

38. Lertpaitoonpan W, Moorman TB, Ong SK. Effect of swine manure on sulfamethazine degradation in aerobic and anaerobic soils. Water Air Soil Pollut. 2015;226(3):1-13.

39. Heise J, Höltge S, Schrader S, Kreuzig R. Chemical and biological characterization of non-extractable sulfonamide residues in soil. Chemosphere. 2006:65(11):2352-7.

40. Kreuzig R, Höltge S. Investigations on the fate of sulfadiazine in manured soil: laboratory experiments and test plot studies. Environ Toxicol Chem. 2005;24(4):771-6.

41. Nowak KM, Miltner A, Gehre M, Schäffer A, Kästner M. Formation and fate of bound residues from microbial biomass during 2,4-d degradation in soil. Environ Sci Technol. 2011:45(3):999-1006.

42. Singh R, Paul D, Jain RK. Biofilms: implications in bioremediation. Trends Microbiol. 2006;14(9):389-97.

43. Bhinu VS. Insight into biofilm-associated microbial life. J Mol Microbiol Biotechnol. 2005;10(1):15-21.

44. Jefferson KK. What drives bacteria to produce a biofilm? FEMS Microbiol Lett. 2004;236(2):163-73.

\section{Submit your manuscript to a SpringerOpen ${ }^{\circ}$ journal and benefit from:}

- Convenient online submission

- Rigorous peer review

- Immediate publication on acceptance

- Open access: articles freely available online

- High visibility within the field

- Retaining the copyright to your article

Submit your next manuscript at springeropen.com 